\title{
KIRILLOV-FRENKEL CHARACTER FORMULA FOR LOOP GROUPS, RADIAL PART AND BROWNIAN SHEET
}

\author{
By MANON DEFOSSEUX \\ Université Paris 5
}

\begin{abstract}
We consider the coadjoint action of a Loop group of a compact group on the dual of the corresponding centrally extended Loop algebra and prove that a Brownian motion in a Cartan subalgebra conditioned to remain in an affine Weyl chamber-which can be seen as a space time conditioned Brownian motion-is distributed as the radial part process of a Brownian sheet on the underlying Lie algebra.
\end{abstract}

1. Introduction. It is a famous result that a real Brownian motion conditioned in Doob's sense to remain positive, is distributed as a Bessel 3 process, that is, as the radial process of a 3-dimensional Brownian motion. More generally, if one considers the adjoint action of a compact Lie group on its associated Lie algebra, the radial part process of a Brownian motion on the Lie algebra is distributed as a Brownian motion on a Cartan subalgebra conditioned to remain in a Weyl chamber (see, for instance, [8] for the unitary group and [10] for any compact groups). In this paper, we consider the coadjoint action of a Loop group of a compact group on the dual of the corresponding centrally extended Loop algebra. It allows us to define the radial part process associated to a Brownian sheet on the underlying semisimple Lie algebra. We prove that it is distributed as a Brownian motion on a Cartan subalgebra conditioned to remain in an affine Weyl chamber.

Let us be more precise. Let $K$ be a connected compact Lie group, $\mathfrak{k}$ its Lie algebra and $\mathfrak{t}$ a Cartan subalgebra. One considers a Weyl chamber in $t$. Then the orbits of $\mathfrak{k}$ under the adjoint action of $K$ are parametrized by the Weyl chamber. Actually for any $x \in \mathfrak{k}$, there exists a unique vector in the Weyl chamber which is in the same orbit as $x$. This vector is called the radial part of $x$. The Lie algebra $\mathfrak{k}$ is equipped with an $\operatorname{Ad}(K)$-invariant scalar product, whose restriction to the Cartan subalgebra is invariant for the action of the Weyl group. If we consider the radial part process of a standard Brownian motion on $\mathfrak{k}$, it is a classical fact that this process is distributed as the projection of the Brownian motion on the Cartan subalgebra $\mathfrak{t}$ - which is a Brownian motion on $\mathfrak{t}$ - conditioned in Doob's sense to remain forever in the Weyl chamber (see [10]). The Kirillov's character formula is at the heart of the connection between the two processes.

Received March 2017; revised April 2018.

MSC2010 subject classifications. 17B67, $60 \mathrm{~J} 65$.

Key words and phrases. Kirillov character formula, loop group, Brownian sheet, radial part, Doob transform, Brownian motion in affine Weyl chamber. 
An affine Lie algebra can be realized as a central extension of a loop algebra $L \mathfrak{k}$. Considering the coadjoint action of the loop group $L K$ on the dual of the centrally extended loop algebra, one defines the radial part of an element of this dual space (see Pressley and Segal [14]). Frenkel established in [6] a Kirillov character type formula in the framework of affine Lie algebras considering a Gaussian measure on the dual of $L \mathfrak{k}$ - basically a Brownian motion on $\mathfrak{k}$. In his approach, the conditional law of a Brownian motion on $\mathfrak{k}$ given its radial part provides a natural measure on the corresponding orbit under the action of $L K$. It highly suggested that one could construct a process on a loop algebra, whose radial part process would be distributed as a projection on a Cartan subalgebra, conditioned to remain in an affine Weyl chamber.

The affine Weyl chamber is a fundamental domain for the action of the Weyl group on the Tits cone. The first difficulty is that there is no Euclidean structure on the Cartan subalgebra of an affine Lie algebra, which would be invariant for the action of the Weyl group. So there is no natural Brownian motion to consider on it. The Kirillov's orbit method, on which we based our intuition, suggests a connection between coadjoint orbits for the action of a Loop group on the dual of an affine Lie algebra and irreducible representations of the affine Lie algebra. Tensor product of irreducible representations of an affine Lie algebra makes appear a drift in the direction of the fundamental weight $\Lambda_{0}$. The idea is to consider a process with such a drift in the direction of $\Lambda_{0}$ - which can be seen as a time component-living in an affine Lie algebra. In this paper, we construct such a process, considering a Brownian sheet on the Lie algebra $\mathfrak{k}$ and prove that the corresponding radial part process is distributed as a projection on a Cartan subalgebra, conditioned to remain forever in an affine Weyl chamber.

The paper is organized as follows. In Section 2, we describe the orbits for the coadjoint action of a Loop group of a compact group on the dual of the associated centrally extended loop algebra. In particular, we define a notion of radial part for this action, which is suitable for our context. In Section 3, we give a first statement of the main result of the paper that will be clarify in Section 7. Section 4 is basically a reformulation of the main results of [6]. In this section, we compute the conditional law of a Brownian motion indexed by $[0,1]$ given the end point of its stochastic exponential and recall how Frenkel proves that this conditional law leads to a Kirillov character formula for affine Lie algebras. In Section 5, we briefly recall the necessary background on affine Lie algebras. In Section 6, we introduce a Brownian motion on a Cartan subalgebra of an affine Lie algebra conditioned-in Doob's sense - to remain forever in an affine Weyl chamber. We prove in Section 7 that this conditioned Doob process has the same law as the radial part process of a Brownian sheet on $\mathfrak{k}$.

\section{Action of loop group and its orbits.}

Loop group and its action. The following presentation is largely inspired by the one given in [14]. Let $K$ be a connected, simply connected, compact Lie group 
and $\mathfrak{k}$ its Lie algebra. By compactness, without loss of generality, we suppose that $K$ is a matrix Lie group. The adjoint action of $K$ on itself, which is denoted by $\operatorname{Ad}$, is defined by $\operatorname{Ad}(k)(u)=k u k^{*}, k, u \in K$. The induced adjoint action of $K$ on its Lie algebra $\mathfrak{k}$ is still denoted by $\operatorname{Ad}$ and is defined by $\operatorname{Ad}(k)(x)=k x k^{*}$, $k \in K, x \in \mathfrak{k}$. The Lie bracket on $\mathfrak{k}$ is denoted by $[\cdot, \cdot]_{\mathfrak{k}}$. We equip $\mathfrak{k}$ with an $\operatorname{Ad}(K)$-invariant inner product $(\cdot, \cdot)$, for instance the negative of the Killing form. We denote by $e$ the identity of $K$. We consider the group $\operatorname{Map}([0,1], K)$ of Borel measurable maps from $[0,1]$ to $K$, the group law being pointwise composition and the subgroup $L K$ of smooth loops from $[0,1]$ to $K$,

$$
L K=\{f:[0,1] \rightarrow K, f \text { is smooth, } f(0)=f(1)\} .
$$

We consider the Lie algebra $L \mathfrak{k}$ over $\mathbb{R}$ of smooth loops from $[0,1]$ to $\mathfrak{k}$, equipped with the Lie bracket given by $[\xi, \eta]_{\mathfrak{k}}$, defined pointwise, for $\xi, \eta \in L \mathfrak{k}$. We define a centrally extended Lie algebra $L \mathfrak{k} \oplus \mathbb{R} c$, where $c$ is an additional formal central element, equipped with a Lie bracket given by

$$
\left[\xi+t_{1} c, \eta+t_{2} c\right]=[\xi, \eta]_{\mathfrak{k}}+\omega(\xi, \eta) c,
$$

for $\xi, \eta \in L \mathfrak{k}, t_{1}, t_{2} \in \mathbb{R}$, where $\omega$ is the cocycle defined by $\omega(\xi, \eta)=\int_{0}^{1}\left(\xi^{\prime}(s)\right.$, $\eta(s)) d s$. A Cartan subalgebra of the extended Loop algebra is $\mathfrak{t} \oplus \mathbb{R} c$, where $\mathfrak{t}$ is identified with the set of $\mathfrak{t}$-valued constant loops. The Lie bracket actually defines a Lie algebra action of $L \mathfrak{k}$ on $L \mathfrak{k} \oplus \mathbb{R} c$ given by

$$
\xi \cdot(\eta+t c)=[\xi, \eta]+\omega(\xi, \eta) c,
$$

for any $\xi \in L \mathfrak{k},(\eta, t) \in L \mathfrak{k} \times \mathbb{R}$. This action comes from the adjoint action of $L K$ on $L \mathfrak{k} \oplus \mathbb{R} c$ defined by

$$
\gamma \cdot(\eta+t c)=\operatorname{Ad}(\gamma)(\eta)+\left(t+\int_{0}^{1}\left(\gamma_{s}^{-1} \gamma_{s}^{\prime}, \eta_{s}\right) d s\right) c,
$$

for any $\gamma \in L K,(\eta, t) \in L \mathfrak{k} \times \mathbb{R}$. The corresponding coadjoint action of $L K$ on the algebraic dual $(L \mathfrak{k} \oplus \mathbb{R} c)^{*}=(L \mathfrak{k})^{*} \oplus \mathbb{R} \Lambda_{0}$, where $\Lambda_{0}(L \mathfrak{k})=0$ and $\Lambda_{0}(c)=1$, is defined by

$$
\gamma \cdot\left(\phi+t \Lambda_{0}\right)=\left[\operatorname{Ad}^{*}(\gamma) \phi-t \int_{0}^{1}\left(\gamma_{s}^{\prime} \gamma_{s}^{-1}, \cdot\right) d s\right]+t \Lambda_{0},
$$

for any $\phi \in(L \mathfrak{k})^{*}, t \in \mathbb{R}$, where $\int_{0}^{1}\left(\gamma_{s}^{\prime} \gamma_{s}^{-1}, \cdot\right) d s$ stands for the linear form defined by

$$
x \in \mathfrak{k} \mapsto \int_{0}^{1}\left(\gamma_{s}^{\prime} \gamma_{s}^{-1}, x_{s}\right) d s
$$


Notice that the coadjoint action of the loop group does not affect the level, that is, the coordinate in $\Lambda_{0}$. Let us equip $L \mathfrak{k}$ with the $L_{2}$-norm, and consider its completion $L_{2}([0,1], \mathfrak{k})$ with respect with the $L_{2}$-norm. We define

$$
\begin{aligned}
L H^{1}([0,1], K)= & \{\gamma:[0,1] \rightarrow K, \gamma \text { is absolutely continuous, } \\
& \left.\gamma^{-1} \gamma^{\prime} \in L_{2}([0,1], \mathfrak{k}), \gamma(0)=\gamma(1)\right\} \\
H^{1}([0,1], \mathfrak{k})= & \{f:[0,1] \rightarrow \mathfrak{k}, f \text { is absolutely continuous, } \\
& \left.f^{\prime} \in L_{2}([0,1], \mathfrak{k}), f(0)=0\right\}, \\
H^{1}([0,1], K)= & \{h:[0,1] \rightarrow K, h \text { is absolutely continuous, } \\
& \left.h^{-1} h^{\prime} \in L_{2}([0,1], \mathfrak{k}), h(0)=e\right\} .
\end{aligned}
$$

Notice that the derivatives are understood in the weak sense. If we denote by $\left(L_{2}([0,1], \mathfrak{k})\right)^{\prime}$ the topological dual of $L_{2}([0,1], \mathfrak{k})$ then $(2)$ defines an action of $L H^{1}([0,1], K)$ on $\left(L_{2}([0,1], \mathfrak{k})\right)^{\prime} \oplus \mathbb{R} \Lambda_{0}$, given by

$$
\gamma \cdot\left(\phi_{x}+t \Lambda_{0}\right)=\int_{0}^{1}\left(\gamma_{s} x_{s}^{\prime} \gamma_{s}^{-1}, \cdot\right) d s-t \int_{0}^{1}\left(\gamma_{s}^{\prime} \gamma_{s}^{-1}, \cdot\right) d s+t \Lambda_{0}
$$

for any $\gamma \in L H^{1}([0,1], K)$, where $\phi_{x}$ is a linear form defined on $L_{2}([0,1], \mathfrak{k})$ by

$$
\phi_{x}(y)=\int_{0}^{1}\left(y_{s}, x_{s}^{\prime}\right) d s
$$

for any $y \in L_{2}([0,1], \mathfrak{k})$, and $x \in H^{1}([0,1], \mathfrak{k})$. This action gives rise to an action of $L H^{1}([0,1], K)$ on $H^{1}([0,1], \mathfrak{k}) \oplus \mathbb{R} \Lambda_{0}$ defined by

$$
\gamma \cdot\left(x+t \Lambda_{0}\right)=\int_{0}^{\cdot}\left(\gamma_{s} x_{s}^{\prime} \gamma_{s}^{-1}-t \gamma_{s}^{\prime} \gamma_{s}^{-1}\right) d s+t \Lambda_{0}
$$

for any $\gamma \in L H^{1}([0,1], K), x \in H^{1}([0,1], \mathfrak{k})$ and $t \in \mathbb{R}$, which satisfies

$$
\phi_{\left(\gamma \cdot\left(x+t \Lambda_{0}\right)-t \Lambda_{0}\right)}+t \Lambda_{0}=\gamma \cdot\left(\phi_{x}+t \Lambda_{0}\right) .
$$

There is another way to make this action appear naturally. One defines a group action of the loop group $L H^{1}([0,1], K)$ on $H^{1}([0,1], K)$ by letting for any $\gamma \in$ $L H^{1}([0,1], K)$,

$$
(\gamma \cdot h)_{s}=\gamma_{0} h_{s} \gamma_{s}^{-1}
$$

for $h \in H^{1}([0,1], K)$ and $s \in[0,1]$. The set $H^{1}([0,1], K)$ is in one-to-one correspondence with the set $H^{1}([0,1], \mathfrak{k})$. Actually for $t \in \mathbb{R}_{+}^{*}$, this is a classical result of differential geometry that for any path $x \in H^{1}([0,1], \mathfrak{k})$ there exists a unique $X \in H^{1}([0,1], K)$ such that $t d X=X d x$. For any $x \in H^{1}([0,1], \mathfrak{k})$, and $t \in \mathbb{R}_{+}^{*}$, one defines $\varepsilon\left(x+t \Lambda_{0}\right)$ as the unique map in $H^{1}([0,1], K)$ which satisfies this differential equation. As

$$
t d\left(\gamma_{0} \varepsilon\left(x+t \Lambda_{0}\right) \gamma^{-1}\right)=\gamma_{0} \varepsilon\left(x+t \Lambda_{0}\right) \gamma^{-1}\left(\gamma d x \gamma^{-1}-t d \gamma \gamma^{-1}\right),
$$


the action of $L H^{1}([0,1], K)$ on $H^{1}([0,1], K)$, and the action of $L H^{1}([0,1], K)$ on $H^{1}([0,1], \mathfrak{k}) \oplus \mathbb{R} \Lambda_{0}$, satisfy

$$
\varepsilon\left(\gamma \cdot\left(x+t \Lambda_{0}\right)\right)=\gamma \cdot \varepsilon\left(x+t \Lambda_{0}\right) .
$$

Roots and weights. We choose a maximal torus $T$ of $K$ and denote by $\mathfrak{t}$ its Lie algebra. We denote by $\mathfrak{g}$ the complexification of $\mathfrak{k}$, that is, $\mathfrak{g}=\mathfrak{k} \oplus i \mathfrak{k}$. We consider the set of real roots

$$
R=\left\{\alpha \in \mathfrak{t}^{*}: \exists X \in \mathfrak{g} \backslash\{0\}, \forall H \in \mathfrak{t},[H, X]=2 i \pi \alpha(H) X\right\} .
$$

We choose a set $\Sigma$ of simple roots of $R$ and denote by $R_{+}$the set of positive roots. The half sum of positive roots is denoted by $\rho$. Letting for $\alpha \in R$,

$$
\mathfrak{g}_{\alpha}=\{X \in \mathfrak{g}: \forall H \in \mathfrak{t},[H, X]=2 i \pi \alpha(H) X\},
$$

the coroot $\alpha^{\vee}$ of $\alpha \in \Sigma$ is defined to be the only vector of $\mathfrak{t}$ in $\left[\mathfrak{g}_{\alpha}, \mathfrak{g}_{-\alpha}\right]$ such that $\alpha\left(\alpha^{\vee}\right)=2$. We denote by $\theta$ the highest root. The dual Coxeter number denoted by $h^{\vee}$ is equal to $1+\rho\left(\theta^{\vee}\right)$, where $\theta^{\vee}$ is the highest coroot. We denote respectively by $Q=\sum_{i} \mathbb{Z} \alpha_{i}$ and $Q^{\vee}=\sum_{i} \mathbb{Z} \alpha_{i}^{\vee}$ the root and the coroot lattice. The weight lattice $\left\{\lambda \in \mathfrak{t}^{*}: \lambda\left(\alpha^{\vee}\right) \in \mathbb{Z}, \forall \alpha \in \Sigma\right\}$ is denoted by $P$ and the set $\left\{\lambda \in \mathfrak{t}^{*}: \lambda\left(\alpha^{\vee}\right) \in\right.$ $\mathbb{N}, \forall \alpha \in \Sigma\}$ of dominant weights is denoted by $P_{+}$.

Orbits and radial part of a path from $H^{1}([0,1], \mathfrak{k})$.

Proposition 2.1. Let $x, y \in H^{1}([0,1], \mathfrak{k})$ and $t \in \mathbb{R}_{+}^{*}$ :

(1) For any $\gamma \in L H^{1}([0,1], K)$, one has $\gamma \cdot\left(x+t \Lambda_{0}\right)=\left(y+t \Lambda_{0}\right)$ if and only if $\gamma \cdot\left(\phi_{x}+t \Lambda_{0}\right)=\phi_{y}+t \Lambda_{0}$.

(2) It exists $\gamma \in L H^{1}([0,1], K)$, such that $\gamma \cdot\left(x+t \Lambda_{0}\right)=\left(y+t \Lambda_{0}\right)$ if and only if there exists $u \in K$ such that $\operatorname{Ad}(u) \varepsilon\left(x+t \Lambda_{0}\right)_{1}=\varepsilon\left(y+t \Lambda_{0}\right)_{1}$.

PROOF. The first point comes from identity (3). For the second, we write that if $\operatorname{Ad}(u) \varepsilon\left(x+t \Lambda_{0}\right)_{1}=\varepsilon\left(y+t \Lambda_{0}\right)_{1}$, and $\gamma=\varepsilon\left(y+t \Lambda_{0}\right)^{-1} u \varepsilon\left(x+t \Lambda_{0}\right)$, then $\gamma \in L H^{1}([0,1], K)$ and $\gamma \cdot\left(x+t \Lambda_{0}\right)=y+t \Lambda_{0}$.

For $\alpha \in \Sigma$ the fundamental reflection $s_{\alpha} \vee$ is defined on $\mathfrak{t}$ by

$$
s_{\alpha^{\vee}}(x)=x-\alpha(x) \alpha^{\vee} \quad \text { for } x \in \mathfrak{t} .
$$

We consider the extended affine Weyl group generated by the reflections $s_{\alpha^{\vee}}$ and the translations by $\alpha^{\vee}, x \in \mathfrak{t} \mapsto x+\alpha^{\vee}$, for $\alpha \in \Sigma$. The fundamental domain for its action on $\mathfrak{t}$ is

$$
A=\left\{x \in \mathfrak{t}: \forall \alpha \in R_{+}, 0 \leq \alpha(x) \leq 1\right\}
$$


(see, for instance, Section 4.8 of [11]). For $x \in K$, one defines $\mathcal{O}_{x}$ as the adjoint orbit through $x$, that is,

$$
\mathcal{O}_{x}=\left\{y \in K: \exists u \in K, y=u x u^{*}\right\} .
$$

We consider the exponential map exp $: \mathfrak{k} \rightarrow K$. As $K$ is simply connected, the set of conjugacy classes $K / \operatorname{Ad}(K)$ is in one-to-one correspondence with the fundamental domain $A$, that is, for all $u \in K$, there exists a unique $x \in A$ such that $u \in \mathcal{O}_{\exp (x)}$ (see [2] for instance). For $t \in \mathbb{R}_{+}$, one defines the alcove $A_{t}$ of level $t$ by

$$
A_{t}=\left\{x \in \mathfrak{t}: \forall \alpha \in R_{+}, 0 \leq \alpha(x) \leq t\right\},
$$

that is, $A_{t}=t A$. Given $t \in \mathbb{R}_{+}^{*}$, every path in $H^{1}([0,1], \mathfrak{k})$ is conjugated to a straight path

$$
s \in[0,1] \mapsto s r
$$

for some $r \in A_{t}$, and one can give the following definition for the radial part of $x \in H^{1}([0,1], \mathfrak{k})$, given a positive level $t \in \mathbb{R}_{+}^{*}$.

Definition 2.2. For $(t, x) \in \mathbb{R}_{+}^{*} \times H^{1}([0,1], \mathfrak{k})$, one defines the radial part of $x+t \Lambda_{0}$ as the unique element $r$ in $A_{t}$ such that $\varepsilon\left(x+t \Lambda_{0}\right)_{1} \in \mathcal{O}_{\exp \left(\frac{r}{t}\right)}$. It is denoted by $\operatorname{rad}\left(x+t \Lambda_{0}\right)$.

Radial part of a continuous semimartingale. The aim of this part is to define the radial part of a $\mathfrak{k}$-valued Brownian path. Such a path is not in $H^{1}([0,1], \mathfrak{k})$ but one can define a stochastic exponential of a Brownian path, which allows us to define its radial part, by analogy with what we have done above. Let $\left(\Omega, \mathcal{F},\left(\mathcal{F}_{S}\right)_{s \in[0,1]}, \mathbb{P}\right)$ be a filtered probability space. The following results can be found for instance in [13] or [9]. If $\left\{x_{s}, s \in[0,1]\right\}$ is an $\mathfrak{k}$-valued continuous semimartingale and $t \in \mathbb{R}_{+}^{*}$, then the stochastic differential equation

$$
t d X=X \circ d x
$$

where o stands for the Stratonovitch integral, has a unique solution starting from $e$. Such a solution is a $K$-valued process, that we still denote by $\left\{\varepsilon\left(x+t \Lambda_{0}\right)_{s}, s \in\right.$ $[0,1]\}$. This is the Stratonovitch stochastic exponential of $\frac{x}{t}$.

DEFINITION 2.3. For $t \in \mathbb{R}_{+}^{*}$, and $x=\left\{x_{s}, s \in[0,1]\right\}$ a continuous $\mathfrak{k}$-valued semimartingale, one defines the radial part of $\left(x+t \Lambda_{0}\right)$ as the unique element $r$ in $A_{t}$ such that $\varepsilon\left(x+t \Lambda_{0}\right)_{1} \in \mathcal{O}_{\exp \left(\frac{r}{t}\right)}$. It is denoted by $\operatorname{rad}\left(x+t \Lambda_{0}\right)$. We extend the definition to $t=0$ letting $\operatorname{rad}\left(x+0 \Lambda_{0}\right)=0$. 
3. Main theorem. In this section, we give a first statement of the main result of the paper. All objects will be defined precisely in the next sections. We consider a standard space-time Brownian motion $\left\{\left(t, b_{t}\right), t \geq 0\right\}$ on $\mathfrak{t}$ and a standard Brownian sheet $\left\{x_{s}^{t}, s \in[0,1], t \geq 0\right\}$ on $\mathfrak{k}$. For any $t, t^{\prime} \in \mathbb{R}_{+}^{*},\left\{\frac{1}{\sqrt{t}} x_{s}^{t}, s \in[0,1]\right\}$ is a standard Brownian motion on $\mathfrak{k}$, and $\left\{x_{s}^{t^{\prime}+t}-x_{s}^{t}, s \in[0,1]\right\}$ is a $\mathfrak{k}$-valued random process independent of $\sigma\left(x_{s}^{r}, s \in[0,1], r \leq t\right)$, having the same law as $\left\{x_{s}^{t^{\prime}}, s \in[0,1]\right\}$. In the sequel we choose a continuous version of it. The definition of the function $\widehat{\varphi}_{d}$ of the theorem is given in Definition 4.5.

THEOREM 3.1. The process $\left\{\left(t, \operatorname{rad}\left(x^{t}+t \Lambda_{0}\right)\right), t \geq 0\right\}$ is a space-time $h$ Doob transform of the space-time Brownian motion $\left\{\left(t, b_{t}\right), t \geq 0\right\}$ killed at the boundary of

$$
\widehat{\mathcal{C}}=\left\{(t, x) \in \mathbb{R}_{+} \times \mathfrak{t}: 0 \leq \alpha(x) \leq t, \alpha \in R_{+}\right\},
$$

with the space-time harmonic function $h=\widehat{\varphi}_{d}$.

The set $\widehat{\mathcal{C}}$ is actually an affine Weyl chamber and the theorem can be seen as an analogue in an affine framework of the fact that the radial part process of a Brownian motion on the Lie algebra $\mathfrak{k}$ is distributed as a Brownian motion on a Cartan subalgebra of $\mathfrak{k}$ conditioned to remain in a Weyl chamber.

\section{Stochastic exponential of a Brownian motion on $\mathfrak{k}$ and Kirillov character} type formula for affine Lie algebras. Frenkel established in [6] a Kirillov character type formula in the framework of affine Lie algebras considering a Brownian motion on $\mathfrak{k}$ and its wrapping on the group $K$. In this section, for the sake of clarity, we give a reformulation of the main results of [6], which fits to our framework.

4.1. The conditional law of a Brownian motion on $\mathfrak{k}$ given the end-point of its stochastic exponential. We denote by $\Lambda$ the kernel of the restriction $\exp _{\mid \mathfrak{t}}$ and by $\Lambda^{*}$ the set of integral weights $\left\{\lambda \in \mathfrak{t}^{*}: \lambda(\Lambda) \subset \mathbb{Z}\right\}$, which is included in $P$ since $\alpha^{\vee} \in \Lambda$ (see [2] for instance). Thus, we define a map $\vartheta_{\lambda}$ on $T$, when $\lambda \in \Lambda^{*}$, by letting $\vartheta_{\lambda}(\exp (x))=e^{2 i \pi \lambda(x)}$, for $x \in \mathfrak{t}$. The irreducible representations of $K$ are parametrized by the set $\Lambda_{+}^{*}=\Lambda^{*} \cap \mathcal{C}^{\vee}$, where $\mathcal{C}^{\vee}=\left\{\lambda \in \mathfrak{t}^{*}: \lambda\left(\alpha^{\vee}\right) \geq 0, \alpha \in \Sigma\right\}$. Here, $K$ is supposed to be simply connected, so that $P=\Lambda^{*}$ and $P_{+}=\Lambda_{+}^{*}$. We denote by $\mathrm{ch}_{\lambda}$ the character of the irreducible representation with highest weight $\lambda \in P_{+}$. We recall that $\mathfrak{t}$ is equipped with a $W$-invariant scalar product $(\cdot, \cdot)$. We identify $\mathfrak{t}$ and $\mathfrak{t}^{*}$ via $(\cdot, \cdot)$ and still denote by $(\cdot, \cdot)$ the scalar product on $\mathfrak{t}^{*}$. We write sometimes $e^{x}$ instead of $\exp (x)$, for $x \in \mathfrak{k}$.

Brownian motion on $K$. Let $\left(\Omega,\left(\mathcal{F}_{S}\right)_{s \in[0,1]}, \mathbb{P}\right)$ be a probability space, where $\left\{\mathcal{F}_{S}, s \in[0,1]\right\}$ is the natural filtration of a $\mathfrak{k}$-valued standard continuous Brownian 
motion $\left\{x_{s}^{\sigma}, s \in[0,1]\right\}$ defined on $\Omega$, with variance $\sigma>0$. In this section, we consider the stochastic exponential $\varepsilon\left(x^{\sigma}+t \Lambda_{0}\right)$ only for $t=1$. Since, for any $t \in \mathbb{R}_{+}^{*}$, we have the identity in law $\varepsilon\left(x^{\sigma}+t \Lambda_{0}\right)=\varepsilon\left(x^{\frac{\sigma}{t^{2}}}+\Lambda_{0}\right)$, there is no loss of generality. In the sequel, we let $\varepsilon\left(x^{\sigma}\right)=\varepsilon\left(x^{\sigma}+\Lambda_{0}\right)$. The stochastic exponential $\left\{\varepsilon\left(x^{\sigma}\right)_{s}, s \in[0,1]\right\}$ is a left Levy process on $K$ starting from $e$, with transition probability $\left\{p_{s}^{\sigma}, s \in[0,1]\right\}$ with respect to the Haar measure on $K$, defined on $K$ by

$$
p_{s}^{\sigma}(x, y)=p_{s}^{\sigma}\left(e, x^{-1} y\right)=\sum_{\lambda \in P_{+}} \operatorname{ch}_{\lambda}(e) \operatorname{ch}_{\lambda}\left(x^{-1} y\right) e^{-\frac{s \sigma(2 \pi)^{2}}{2}\left(\|\lambda+\rho\|^{2}-\|\rho\|^{2}\right)},
$$

$s \in[0,1], x, y \in K$ (see, for instance, [5]). In the sequel, we write $p_{s}^{\sigma}(x)$ instead of $p_{s}^{\sigma}(e, x)$. This process is a Brownian motion on $K$. The following proposition states a Girsanov formula for a Brownian motion on a compact Lie group. It is proved for instance in [3,7] or [13]. For a $\mathfrak{k}$-valued $L_{2}$ function $y$, and a $\mathfrak{k}$-valued continuous semimartingale $\left\{x_{s}, s \in[0,1]\right\}, \int_{0}^{t}\left(y_{s}, d x_{s}\right)$ is defined as the stochastic integral of $y$ with respect to $x$, for any $t \in[0,1]$.

THEOREM 4.1. Let $\left\{x_{s}^{\sigma}, s \in[0,1]\right\}$ be a Brownian motion on $\mathfrak{k}$, with variance $\sigma \in \mathbb{R}_{+}^{*}$, and $h \in H^{1}([0,1], K)$. If $\mu^{\sigma}$ is the law of $\left\{\varepsilon\left(x^{\sigma}\right)_{s}, s \in[0,1]\right\}$, then

$$
\frac{d\left(\mathcal{R}_{h}\right)_{*} \mu^{\sigma}}{d \mu^{\sigma}}=e^{\frac{1}{\sigma} \int_{0}^{1}\left(h_{s}^{-1} h_{s}^{\prime}, d x_{s}^{\sigma}\right)-\frac{1}{2 \sigma} \int_{0}^{1}\left(h_{s}^{-1} h_{s}^{\prime}, h_{s}^{-1} h_{s}^{\prime}\right) d s},
$$

where $\left(\mathcal{R}_{h}\right)_{*} \mu^{\sigma}$ is the law of $\left\{\varepsilon\left(x^{\sigma}\right)_{s} h_{s}, s \in[01]\right\}$.

For $z \in K$, we write $\mathbb{P}^{z}$, for the probability defined on $\mathcal{F}_{1}$ as the conditional probability $\mathbb{P}\left(\cdot \mid \varepsilon\left(x^{\sigma}\right)_{1}=z\right)$. One has for any $s \in(0,1)$,

$$
\mathbb{P}_{\mid \mathcal{F}_{s}}^{z}=\frac{p_{1-s}^{\sigma}\left(\varepsilon\left(x^{\sigma}\right)_{s}, z\right)}{p_{1}(z)} \cdot \mathbb{P}_{\mid \mathcal{F}_{s}} .
$$

Note that under $\mathbb{P}^{z},\left\{x_{s}^{\sigma}, s \in[0,1)\right\}$ remains a continuous semimartingale (see, for instance, [1], Theorem 14), so that the stochastic integral $\int_{0}^{t}\left(y_{s}, d x_{s}^{\sigma}\right)$ is welldefined under $\mathbb{P}^{z}$, for any $\mathfrak{k}^{k}$-valued $L_{2}$ function $y$ and $t \in(0,1)$. Theorem $4.1 \mathrm{im}$ plies the following proposition, which appears in Proposition (5.2.12) of [6].

Proposition 4.2. Let $\left\{x_{s}^{\sigma}, s \in[0,1]\right\}$ be a standard Brownian motion on $\mathfrak{k}$

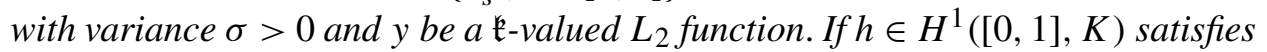
$h^{-1} h^{\prime}=y$, then for any $t \in(0,1)$,

$$
e^{-\frac{1}{2 \sigma} \int_{0}^{t}\left(y_{s}, y_{s}\right) d s} \mathbb{E}\left(e^{\frac{1}{\sigma} \int_{0}^{t}\left(y_{s}, d x_{s}^{\sigma}\right)} \mid \varepsilon\left(x^{\sigma}\right)_{1}=z\right)=\frac{p_{1}^{\sigma}\left(z h_{t}^{-1}\right)}{p_{1}^{\sigma}(z)} .
$$


ProOF. For $t \in(0,1)$,

$$
\begin{aligned}
& \mathbb{E}\left(e^{\frac{1}{\sigma} \int_{0}^{t}\left(y_{s}, d x_{s}^{\sigma}\right)} \mid \varepsilon\left(x^{\sigma}\right)_{1}=z\right) \\
& \quad=\mathbb{E}\left(e^{\frac{1}{\sigma} \int_{0}^{t}\left(y_{s}, d x_{s}^{\sigma}\right)} \frac{p_{1-t}^{\sigma}\left(\varepsilon\left(x^{\sigma}\right)_{t}, z\right)}{p_{1}^{\sigma}(z)}\right) \\
& \quad=e^{\frac{1}{2 \sigma} \int_{0}^{t}\left(y_{s}, y_{s}\right) d s} \mathbb{E}\left(e^{\frac{1}{\sigma} \int_{0}^{t}\left(y_{s}, d x_{s}^{\sigma}\right)-\frac{1}{2 \sigma} \int_{0}^{t}\left(y_{s}, y_{s}\right) d s} \frac{p_{1-t}^{\sigma}\left(\varepsilon\left(x^{\sigma}\right)_{t}, z\right)}{p_{1}^{\sigma}(z)}\right) .
\end{aligned}
$$

Let $h \in H^{1}(K)$ such that $h^{-1} h^{\prime}=y$. Theorem 4.1 implies that

$$
\begin{aligned}
\mathbb{E}\left(e^{\frac{1}{\sigma} \int_{0}^{t}\left(y_{s}, d x_{s}^{\sigma}\right)-\frac{1}{2 \sigma} \int_{0}^{t}\left(y_{s}, y_{s}\right) d s} \frac{p_{1-t}^{\sigma}\left(\varepsilon\left(x^{\sigma}\right)_{t}, z\right)}{p_{1}^{\sigma}(z)}\right) \\
=\mathbb{E}\left(\frac{p_{1-t}^{\sigma}\left(\varepsilon\left(x^{\sigma}\right)_{t} h_{t}, z\right)}{p_{1}^{\sigma}(z)}\right) \\
=\mathbb{E}\left(\frac{p_{1-t}^{\sigma}\left(\varepsilon\left(x^{\sigma}\right)_{t}, z h_{t}^{-1}\right)}{p_{1}^{\sigma}(z)}\right) \\
=\frac{p_{1}^{\sigma}\left(z h_{t}^{-1}\right)}{p_{1}^{\sigma}(z)}
\end{aligned}
$$

which gives the proposition.

The following lemma has been proved in [6].

LEMMA 4.3. For $k_{1}, k_{2} \in K, s \in[0,1]$,

$$
\int_{K} p_{s}^{\sigma}\left(k_{1}, u k_{2} u^{*}\right) d u=\sum_{\lambda \in P_{+}} \operatorname{ch}_{\lambda}\left(k_{1}^{-1}\right) \operatorname{ch}_{\lambda}\left(k_{2}\right) e^{-\frac{s \sigma(2 \pi)^{2}}{2}\left(\|\rho+\lambda\|^{2}-\|\rho\|^{2}\right)} .
$$

The previous lemma and Proposition 4.2 imply the following one.

Proposition 4.4. Let $\left\{x_{s}^{\sigma}, s \in[0,1]\right\}$ be a standard Brownian motion on $\mathfrak{k}$ starting from 0 , with variance $\sigma>0$. For $y$ a $\mathfrak{k}$-valued $L_{2}$ function, $r \in A$, and $t \in(0,1)$,

$$
\begin{aligned}
& e^{-\frac{1}{2 \sigma} \int_{0}^{t}\left(y_{s}, y_{s}\right) d s} \mathbb{E}\left(e^{\frac{1}{\sigma} \int_{0}^{t}\left(y_{s}, d x_{s}^{\sigma}\right)} \mid \operatorname{rad}\left(x^{\sigma}\right)=r\right) \\
& =\frac{1}{p_{1}^{\sigma}\left(e^{r}\right)} \sum_{\lambda \in P_{+}} \operatorname{ch}_{\lambda}\left(h_{t}^{-1}\right) \operatorname{ch}_{\lambda}\left(e^{r}\right) e^{-\frac{\sigma(2 \pi)^{2}}{2}\left(\|\rho+\lambda\|^{2}-\|\rho\|^{2}\right)},
\end{aligned}
$$

where $h \in H^{1}([0,1], K)$ satisfies $h^{-1} h^{\prime}=y$. 
PROOF.

$$
\begin{aligned}
\mathbb{E}\left(e^{\frac{1}{\sigma} \int_{0}^{t}\left(y_{s}, d x_{s}^{\sigma}\right) d s} \mid \operatorname{rad}\left(x^{\sigma}\right)=r\right) & =\mathbb{E}\left(\mathbb{E}\left(e^{\frac{1}{\sigma} \int_{0}^{t}\left(y_{s}, d x_{s}^{\sigma}\right) d s} \mid \varepsilon\left(x^{\sigma}\right)_{1}\right) \mid \operatorname{rad}\left(x^{\sigma}\right)=r\right) \\
& =e^{\frac{1}{2 \sigma} \int_{0}^{t}\left(y_{s}, y_{s}\right) d s} \mathbb{E}\left(\frac{p_{1}^{\sigma}\left(\varepsilon\left(x^{\sigma}\right)_{1} h_{t}^{-1}\right)}{p_{1}^{\sigma}\left(\varepsilon\left(x^{\sigma}\right)_{1}\right)} \mid \operatorname{rad}\left(x^{\sigma}\right)=r\right) \\
& =e^{\frac{1}{2 \sigma} \int_{0}^{t}\left(y_{s}, y_{s}\right) d s} \int_{K} \frac{p_{1}^{\sigma}\left(u e^{r} u^{*} h_{t}^{-1}\right)}{p_{1}^{\sigma}\left(e^{r}\right)} d u,
\end{aligned}
$$

which proves the proposition thanks to Lemma 4.3.

4.2. A Kirillov character type formula for affine Lie algebras. Let $\left\{x_{s}^{\sigma}, s \in\right.$ $[0,1]\}$ be a Brownian motion on $\mathfrak{k}$, with variance $\sigma>0$, and its stochastic exponential $\left\{\varepsilon\left(x^{\sigma}\right)_{s}, s \in[0,1]\right\}$. In the sequel, we let, for $x \in \mathfrak{t}$,

$$
\pi(x)=\prod_{\alpha \in R_{+}}\left(e^{i \pi \alpha(x)}-e^{-i \pi \alpha(x)}\right) \quad \text { and } \quad h(x)=\prod_{\alpha \in R_{+}} \alpha(x) .
$$

Definition 4.5. For $x \in \mathfrak{t}, y \in \mathfrak{t} \oplus i \mathfrak{t}, \sigma \in \mathbb{R}_{+}^{*}$, we let

$$
\begin{aligned}
\widehat{\varphi}_{d+y} & \left(\frac{1}{\sigma}, \frac{x}{\sigma}\right) \\
& =\sigma^{n / 2} e^{\frac{1}{2 \sigma}(y, y)+\frac{1}{2 \sigma}(x, x)} \sum_{\mu \in P_{+}} \pi(x) \operatorname{ch}_{\mu}\left(e^{x}\right) \operatorname{ch}_{\mu}\left(e^{-y}\right) e^{-\frac{\sigma}{2}(2 \pi)^{2}\|\mu+\rho\|^{2}} .
\end{aligned}
$$

A Kirillov character type formula for affine Lie algebras has been proved by Frenkel in [6]. It can be formulated as in the following theorem, which is a consequence of propositions 4.4. This is the analogue of the Harish-Chandra formula.

THEOREM 4.6. For $y \in L_{2}([0,1], \mathfrak{k})$, and $z \in A$, one has for $t \in(0,1)$,

$$
\mathbb{E}\left(e^{\frac{1}{\sigma} \int_{0}^{t}\left(y_{s}, d x_{s}^{\sigma}\right)} \mid \operatorname{rad}\left(x^{\sigma}\right)=z\right)=e^{\frac{1}{2 \sigma} \int_{0}^{t}\left(y_{s}, y_{s}\right) d s} e^{-\frac{1}{2 \sigma}(a, a)} \frac{\widehat{\varphi}_{d+a}\left(\frac{1}{\sigma}, \frac{z}{\sigma}\right)}{\widehat{\varphi}_{d}\left(\frac{1}{\sigma}, \frac{z}{\sigma}\right)},
$$

where $h^{-1} h^{\prime}=y$ and $h_{t} \in \mathcal{O}_{e^{a}}$, with $a \in \mathfrak{t}$. In particular for $y \in \mathfrak{t}$, one has

$$
\mathbb{E}\left(e^{\frac{1}{\sigma}\left(y, x_{1}^{\sigma}\right)} \mid \operatorname{rad}\left(x^{\sigma}\right)=z\right)=\frac{\widehat{\varphi}_{d+y}\left(\frac{1}{\sigma}, \frac{z}{\sigma}\right)}{\widehat{\varphi}_{d}\left(\frac{1}{\sigma}, \frac{z}{\sigma}\right)} .
$$

REMARK 4.7. Let us make some nonrigorous remarks about these formulae. Previously, we have considered the identification of $L_{2}([0,1], \mathfrak{k})^{\prime} \oplus \mathbb{R} \Lambda_{0}$ with $H_{1}([0,1], \mathfrak{k}) \oplus \mathbb{R} \Lambda_{0}$ letting $\varphi_{x}=\int_{0}^{1}\left(\cdot, x_{s}^{\prime}\right) d s$ for $x \in H_{1}([0,1], \mathfrak{k})$. In the first formula, the stochastic integral $\int_{0}^{1}\left(\cdot, d x_{s}^{\sigma}\right)$ can be seen as a random linear functional. Its conditional law given $\varepsilon\left(x^{\sigma}\right) \in \mathcal{O}_{e(z)}$ has to be thought as a measure 
on a coadjoint orbit through $\varphi_{\pi_{z}}+\Lambda_{0}$ where $\pi_{z}$ is the straight path $\pi_{z}(s)=s z$, $s \in[0,1]$. For the second formula, we notice that the restriction of $\varphi_{x}$ to $\mathfrak{t}$ is equal to $y \in \mathfrak{t} \mapsto\left(y, x_{1}\right)$. Thus the law of $x_{1}^{\sigma}$ given $\varepsilon\left(x^{\sigma}\right) \in \mathcal{O}_{e(z)}$ has to be thought as a Duistermaat-Heckman distribution associated to $\varphi_{\pi_{z}}+\Lambda_{0}$.

5. Affine Lie algebras. In this part, we consider an affine Lie algebra whose Cartan matrix is an extended Cartan matrix of the simple finite dimensional complex Lie algebra $\mathfrak{g}$. Such an algebra is a nontwisted affine Lie algebra. It can be realized as a central extension of the Lie algebra of Laurent polynomials with coefficients in $\mathfrak{g}$. For our purpose, we only need to consider a realization of its Cartan subalgebra. If $\left\{\alpha_{1}, \ldots, \alpha_{n}\right\}$ and $\left\{\alpha_{1}^{\vee}, \ldots, \alpha_{n}^{\vee}\right\}$ are respectively the sets of simple real roots and coroots of the group $K$ previously considered, we let

$$
\mathfrak{h}=\operatorname{span}_{\mathbb{C}}\left\{\alpha_{0}^{\vee}=c-\theta^{\vee}, \alpha_{1}^{\vee}, \ldots, \alpha_{n}^{\vee}, d\right\}
$$

and

$$
\mathfrak{h}^{*}=\operatorname{span}_{\mathbb{C}}\left\{\alpha_{0}=\delta-\theta, \alpha_{1}, \ldots, \alpha_{n}, \Lambda_{0}\right\}
$$

where

$$
\alpha_{i}(d)=\delta_{i 0}, \quad \delta\left(\alpha_{i}^{\vee}\right)=0, \quad \Lambda_{0}\left(\alpha_{i}^{\vee}\right)=\delta_{i 0}, \quad \Lambda_{0}(d)=0 .
$$

The Killing form on $\mathfrak{k}$ is from now on normalized such that $\left(\theta^{\vee}, \theta^{\vee}\right)=2$. We consider its restriction to $\mathfrak{t}$ and extend it to $\mathfrak{h}$ by $\mathbb{C}$-linearity, and by letting

$$
(\mathbb{C} c+\mathbb{C} d, \mathfrak{t})=0, \quad(c, c)=(d, d)=0, \quad(c, d)=1 .
$$

The following definitions mainly come from Chapters 1 and 6 of [12]. The linear isomorphism

$$
\begin{aligned}
v: \mathfrak{h} & \rightarrow \mathfrak{h}^{*}, \\
h & \mapsto(h, \cdot)
\end{aligned}
$$

identifies $\mathfrak{h}$ and $\mathfrak{h}^{*}$. We still denote by $(\cdot, \cdot)$ the induced bilinear form on $\mathfrak{h}^{*}$. We record that

$$
\left(\delta, \alpha_{i}\right)=0, \quad i=0, \ldots, n, \quad(\delta, \delta)=0, \quad\left(\delta, \Lambda_{0}\right)=1 .
$$

Notice that here $v\left(\theta^{\vee}\right)=\theta$ and $\left(\theta^{\vee} \mid \theta^{\vee}\right)=(\theta \mid \theta)=2$. One defines the Weyl group $\widehat{W}$, as the subgroup of $G L\left(\mathfrak{h}^{*}\right)$ generated by fundamental reflections $s_{\alpha}, \alpha \in \Pi$, defined by

$$
s_{\alpha}(\beta)=\beta-\beta\left(\alpha^{\vee}\right) \alpha, \quad \beta \in \mathfrak{h}^{*} .
$$

Under the identification of $\mathfrak{h}$ and $\mathfrak{h}^{*}$, the action of the affine Weyl group on $\mathfrak{h}$ is defined by $w x=v^{-1} w v x, x \in \mathfrak{h}, w \in \widehat{W}$. The form $(\cdot, \cdot)$ is $\widehat{W}$-invariant. The affine 


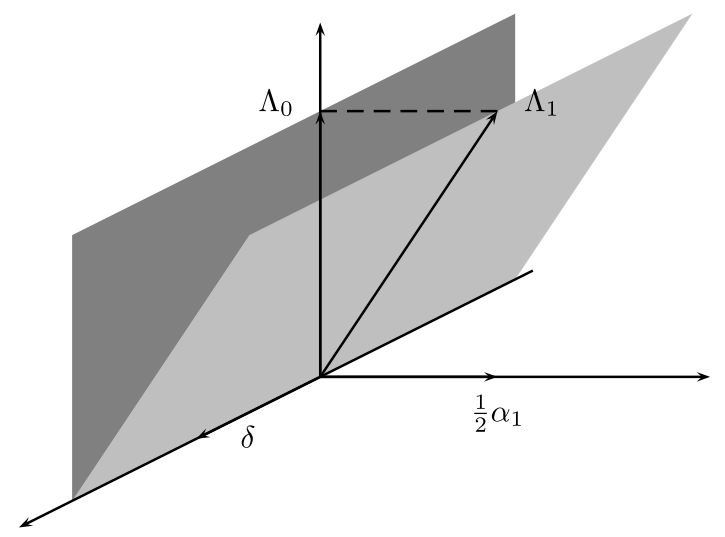

FIG. 1. The affine Weyl Chamber corresponding to $A_{1}^{(1)}$.

Weyl group $\widehat{W}$ is the semidirect product $W \ltimes \Gamma$ (Proposition 6.5, Chapter 6 of [12]) where $\Gamma$ is the group of transformations $t_{\gamma}, \gamma \in v\left(Q^{\vee}\right)$, defined by

$$
t_{\gamma}(\lambda)=\lambda+\lambda(c) \gamma-\left((\lambda, \gamma)+\frac{1}{2}(\gamma, \gamma) \lambda(c)\right) \delta, \quad \lambda \in \mathfrak{h}^{*} .
$$

Thus, if $w \in W, \gamma \in v\left(Q^{\vee}\right)$, and $\lambda \in \mathfrak{h}^{*}$,

$$
w t_{\gamma}(\lambda)=w(\lambda+\lambda(c) \gamma)-\left((\lambda, \gamma)+\frac{1}{2}(\gamma, \gamma) \lambda(c)\right) \delta \text {. }
$$

The Weyl chamber $\widehat{\mathcal{C}}$ is defined by

$$
\widehat{\mathcal{C}}=\left\{x \in \mathfrak{h}: \forall i \in\{0, \ldots, n\}, \alpha_{i}(x) \geq 0\right\} .
$$

As the highest root $\theta$ is equal to $\delta-\alpha_{0}$, one has for any $t \in \mathbb{R}_{+}, y \in \mathfrak{t}$

$$
(t d+y) \in \widehat{\mathcal{C}} \Leftrightarrow \forall \alpha \in R_{+}, 0 \leq \alpha(y) \leq t .
$$

Thus in the sequel we identify $\widehat{\mathcal{C}}$ with the subspace of $\mathbb{R} \times \mathfrak{t}$

$$
\left\{(t, x) \in \mathbb{R}_{+} \times \mathfrak{t}: 0 \leq \alpha(x) \leq t, \alpha \in R_{+}\right\} .
$$

The affine Weyl chamber $\widehat{\mathcal{C}}$ for the $A_{1}^{(1)}$ type is drawn in Figure 1: it is the area delimited by dark gray and gray half-planes. The alcove $A$ is the dashed interval.

\section{A space-time Brownian motion on $\mathfrak{t}$ conditioned to remain in the affine} Weyl chamber. Let us consider a standard Brownian motion $\left\{b_{t}, t \geq 0\right\}$ on $\mathfrak{t}$ and a space time Brownian motion $\left\{\left(\tau_{t}, b_{t}\right), t \geq 0\right\}$. For $u \in \mathbb{R}, x \in \mathfrak{t}$, we denote by $\mathbb{W}_{u, x}$ a probability under which $\tau_{t}=u+t$, for all $t \geq 0$, and $\left\{b_{t}, t \geq 0\right\}$ is a standard Brownian motion starting from $x$. Let us consider the stopping time

$$
T=\inf \left\{t \geq 0:\left(\tau_{t}, b_{t}\right) \notin \widehat{\mathcal{C}}\right\}
$$


LEMMA 6.1. For $(u, x)$ in the interior of $\widehat{\mathcal{C}}$, and $y \in \mathfrak{t} \oplus i \mathfrak{t}$, the process

$$
\left\{e^{-\frac{(y, y) t \wedge T}{2}} \widehat{\varphi}_{d+y}\left(\tau_{t \wedge T}, b_{t \wedge T}\right), t \geq 0\right\}
$$

is a martingale under $\mathbb{W}_{u, x}$.

ProOF. One has for $(t, z) \in \mathbb{R}_{+}^{*} \times \mathfrak{t}$,

$$
e^{-\frac{t}{2}(y, y)} \widehat{\varphi}_{d+y}(t, z)=\sum_{\mu \in P_{+}} \frac{1}{t^{n / 2}} e^{\frac{1}{2 t}(z, z)} \pi\left(\frac{z}{t}\right) \operatorname{ch}_{\mu}\left(e^{\frac{z}{t}}\right) \operatorname{ch}_{\mu}\left(e^{-y}\right) e^{-\frac{1}{2 t}(2 \pi)^{2}\|\mu+\rho\|^{2}},
$$

where, by the Weyl's character formula for compact Lie groups

$$
\operatorname{ch}_{\lambda}\left(e^{z}\right)=\frac{1}{\pi(z)} \sum_{w \in W}(-1)^{w} e^{2 i \pi\langle w(\lambda+\rho), z\rangle} .
$$

Choose an orthonormal basis $v_{1}, \ldots, v_{n}$ of $\mathfrak{t}$ and consider for $\mu \in P_{+}$, a function $g_{\mu}$ defined on $\mathbb{R}_{+}^{*} \times \mathbb{R}^{n}$ by

$$
g_{\mu}\left(t, z_{1}, \ldots, z_{n}\right)=\frac{1}{t^{n / 2}} e^{\frac{1}{2 t}(z, z)} \pi\left(\frac{z}{t}\right) \operatorname{ch}_{\mu}\left(e^{\frac{z}{t}}\right) \operatorname{ch}_{\mu}\left(e^{-y}\right) e^{-\frac{1}{2 t}(2 \pi)^{2}\|\mu+\rho\|^{2}},
$$

where $z=z_{1} v_{1}+\cdots+z_{n} v_{n}$. Letting $\Delta=\sum_{i=1}^{n} \partial_{z_{i} z_{i}}$, the function $g_{\mu}$ satisfies

$$
\left(\frac{1}{2} \Delta+\partial_{t}\right) g_{\mu}=0
$$

As the sums $\sum \Delta g_{\mu}$ and $\sum \partial_{t} g_{\mu}$ are normally convergent on any compact subsets of $\mathbb{R}_{+}^{*} \times \mathbb{R}^{n}$, one has

$$
\left(\frac{1}{2} \Delta+\partial_{t}\right) \sum_{\mu \in \mathbb{P}_{+}} g_{\mu}=0
$$

on $\mathbb{R}_{+}^{*} \times \mathbb{R}^{n}$, which implies that $\left\{e^{-\frac{(y, y) t \wedge T}{2}} \widehat{\varphi}_{d+y}\left(\tau_{t \wedge T}, b_{t \wedge T}\right), t \geq 0\right\}$ is a local martingale. As

$$
(t, z) \in \mathbb{R}_{+}^{*} \times \mathbb{R}^{n} \mapsto e^{-\frac{(y, y) t}{2}} \widehat{\varphi}_{d+y}(t, z)
$$

is bounded on any compact subsets of $\mathbb{R}_{+}^{*} \times \mathbb{R}^{n},\left\{e^{-\frac{(y, y) t \wedge T}{2}} \widehat{\varphi}_{d+y}\left(\tau_{t \wedge T}, b_{t \wedge T}\right), t \geq\right.$ $0\}$ is a martingale under $\mathbb{W}_{u, x}$.

LEMMA 6.2. Let $t>0$. If $(t, x) \in \widehat{\mathcal{C}}$, then $\widehat{\varphi}_{d}(t, x) \geq 0$, with equality if and only if $(t, x)$ is on the boundary of $\widehat{\mathcal{C}}$.

PROOF. For any $z \in \mathfrak{t}$, and $t>0$,

$$
\widehat{\varphi}_{d}(t, z)=C \times p_{1}^{\frac{1}{t}}\left(e^{\frac{z}{t}}\right) \pi\left(\frac{z}{t}\right) e^{\frac{1}{2 t}\|z\|^{2}-\frac{1}{2 t}(2 \pi)^{2}\|\rho\|^{2}},
$$


where $C$ is a positive constant, which ensures in particular that

$$
\widehat{\varphi}_{d}(t, z) \geq 0
$$

for $z \in A_{t}$, with equality if and only if $z \in \partial A_{t}$.

Let $\left\{\mathcal{F}_{t}, t \geq 0\right\}$ be the natural filtration of $\left\{\left(t, b_{t}\right), t \geq 0\right\}$. As for $(u, x)$ in the interior of $\widehat{\mathcal{C}},\left\{\widehat{\varphi}_{d}\left(\tau_{t \wedge T}, b_{t \wedge T}\right), t \geq 0\right\}$ is a positive martingale under $\mathbb{W}_{u, x}$ such that $\widehat{\varphi}_{d}\left(\tau_{T}, b_{T}\right)=0$, one defines a measure $\mathbb{Q}_{u, x}$ on $\mathcal{F}_{\infty}$ as below.

Definition 6.3. Let $u>0$ and $x \in \mathfrak{t}$ such that $(u, x)$ is in the interior of $\widehat{\mathcal{C}}$. One defines a probability $\mathbb{Q}_{u, x}$ on $\mathcal{F}_{\infty}$ letting

$$
\mathbb{Q}_{u, x}(B)=\mathbb{E}_{\mathbb{W}_{u, x}}\left(\frac{\widehat{\varphi}_{d}\left(\tau_{t}, b_{t}\right)}{\widehat{\varphi}_{d}(u, x)} 1_{T \geq t, B}\right) \quad \text { for } B \in \mathcal{F}_{t}, t \geq 0 .
$$

Lemma 6.1 and the fact that for $y \in \mathfrak{t} \oplus i \mathfrak{t}, \widehat{\varphi}_{d+y}\left(\tau_{T}, b_{T}\right)=0$ imply immediately the following proposition.

Proposition 6.4. For $r, t \in \mathbb{R}_{+}^{*},(u, x)$ in the interior of $\widehat{\mathcal{C}}$, and $y \in \mathfrak{t} \oplus i \mathfrak{t}$, one has

$$
\mathbb{E}_{\mathbb{Q}_{u, x}}\left(\frac{\widehat{\varphi}_{d+y}\left(\tau_{t}, b_{t}\right)}{\widehat{\varphi}_{d}\left(\tau_{t}, b_{t}\right)}\right)=\frac{\widehat{\varphi}_{d+y}(u, x)}{\widehat{\varphi}_{d}(u, x)} e^{\frac{(y, y)}{2} t}
$$

and

$$
E_{\mathbb{Q}_{u, x}}\left(\frac{\widehat{\varphi}_{d+y}\left(\tau_{r+t}, b_{t+r}\right)}{\widehat{\varphi}_{d}\left(\tau_{t+r}, b_{t+r}\right)} \mid \mathcal{F}_{r}\right)=\frac{\widehat{\varphi}_{d+y}\left(\tau_{r}, b_{r}\right)}{\widehat{\varphi}_{d}\left(\tau_{r}, b_{r}\right)} e^{\frac{(y, y)}{2} t} .
$$

\section{Conditioned space time Brownian motion and radial part of a Brownian} sheet. In this last section, we prove the main result of the paper which states that the conditioned Doob process in an affine Weyl chamber introduced in Section 6, has the same law as the radial part process of a Brownian sheet on $\mathfrak{k}$. It is stated in Theorem 7.8. The proof rests on an intertwining proved in Proposition 7.9, which allows us to apply a Rogers and Pitman's criterion. Let $\left\{x_{s}^{t}, s \in[0,1], t \geq 0\right\}$ be a standard Brownian sheet on $\mathfrak{k}$. For any $t, t^{\prime} \in \mathbb{R}_{+}^{*},\left\{\frac{1}{\sqrt{t}} x_{s}^{t}, s \in[0,1]\right\}$ is a standard Brownian motion on $\mathfrak{k}$, and $\left\{x_{s}^{t^{\prime}+t}-x_{s}^{t}, s \in[0,1]\right\}$ is a $\mathfrak{k}$-valued random process independent of $\sigma\left(x_{s}^{r}, s \in[0,1], r \leq t\right)$, having the same law as $\left\{x_{s}^{t^{\prime}}, s \in[0,1]\right\}$. In the sequel, we choose a continuous version of it. We denote by $C([0,1], \mathfrak{k})$ the set of continuous maps from $[0,1]$ to $\mathfrak{k}$. Proposition 7.4 and Corollary 7.5 prove the existence of an entrance law for the conditioned process in the affine Weyl chamber introduced in Section 6, and the entrance point 0. 
LEMMA 7.1. For any $y \in \mathfrak{t} \oplus i \mathfrak{t}$,

$$
\frac{\widehat{\varphi}_{d+y}(u, x)}{\widehat{\varphi}_{d}(u, x)}
$$

converges toward 1 when $(u, x)$ goes to 0 within the affine Weyl chamber. In the sequel, we write $\frac{\widehat{\varphi}_{d+y}(0,0)}{\widehat{\varphi}_{d}(0,0)}=1$.

PROOF. One has for $(u, x)$ in the interior of $\widehat{\mathcal{C}}$

$$
\widehat{\varphi}_{d+y}(u, x)=\frac{1}{u^{n / 2}} e^{\frac{u}{2}(y, y)+\frac{1}{2 u}(x, x)} \pi\left(\frac{x}{u}\right) \sum_{\mu \in P_{+}} \operatorname{ch}_{\mu}\left(e^{\frac{x}{u}}\right) \operatorname{ch}_{\mu}\left(e^{-y}\right) e^{-\frac{1}{2 u}(2 \pi)^{2}\|\mu+\rho\|^{2}},
$$

Lemma 13.13 of [12] implies that the dominant term in the sum of the right-hand side of the identity is $e^{-\frac{1}{2 u}(2 \pi)^{2}\|\rho\|^{2}}$, and the proposition follows.

LEMMA 7.2. Let us fix $t>0$. If $\mu_{u, x}$ is the law of $\frac{b_{t}}{t+u}$ under $\mathbb{Q}_{u, x}$ then for any $y \in \mathfrak{t} \oplus i \mathfrak{t}$,

$$
\int_{A} \frac{\widehat{\varphi}_{d+y}(t, t z)}{\widehat{\varphi}_{d}(t, t z)} \mu_{u, x}(d z)
$$

converges toward $e^{t \frac{(y, y)}{2}}$ when $(u, x)$ goes to 0 within the affine Weyl chamber.

PROOF. If $\mu_{u, x}$ is the law of $\frac{b_{t}}{t+u}$ under $\mathbb{Q}_{u, x}$ then

$$
\int_{A} \frac{\widehat{\varphi}_{d+y}(t+u,(t+u) z)}{\widehat{\varphi}_{d}(t+u,(t+u) z)} d \mu_{u, x}(z)=\frac{\widehat{\varphi}_{d+y}(u, x)}{\widehat{\varphi}_{d}(u, x)} e^{\frac{t}{2}(y, y)} .
$$

The Weyl dimension formula gives that $\left|\operatorname{ch}_{\mu}\left(e^{z}\right)\right| \leq \frac{h(\mu+\rho)}{h(\rho)}$ which implies that $\frac{\widehat{\varphi}_{d+y}((t+u),(t+u) z)}{\widehat{\varphi}_{d}(t+u,(t+u) z)}$ converges to $\frac{\widehat{\varphi}_{d+y}(t, t z)}{\widehat{\varphi}_{d}(t, t z)}$, uniformly in $z \in A$, when $u$ goes to 0 . Thus the lemma follows from Lemma 7.1

The following proposition follows immediately from theorem (4.3.4) of [6].

Proposition 7.3. For $x \in \mathfrak{t}, y \in \mathfrak{t} \oplus i \mathfrak{t}, t \in \mathbb{R}_{+}^{*}$, one has

$$
\widehat{\varphi}_{d+y}(t, x)=\frac{k}{\pi(y)} \sum_{\gamma \in Q^{\vee}} \sum_{w \in W}(-1)^{w} e^{(w(x+t \gamma), y)} e^{-(x, \gamma)-\frac{1}{2}(\gamma, \gamma) t},
$$

where $k$ is a constant which doesn't depend on $x, y$ and $t$.

Proposition 7.4. Under $\mathbb{Q}_{u, x}$, for $t>0, b_{t}$ converges in law toward the radial part of $\left\{t \Lambda_{0}+x_{s}^{t}, s \in[0,1]\right\}$, when $(u, x)$ goes to 0 within the affine Weyl chamber. 
PROOF. As $\left\{\frac{1}{t} x_{s}^{t}, s \in[0,1]\right\}$ is a standard Brownian motion on $\mathfrak{k}$ with variance $\frac{1}{t}$, Theorem 4.6 gives for $z \in A, y \in \mathfrak{t} \oplus i \mathfrak{t}$,

$$
\mathbb{E}\left(e^{\left(x_{1}^{t}, y\right)} \mid \operatorname{rad}\left(\left\{\frac{x_{s}^{t}}{t}, s \in[0,1]\right\}\right)=z\right)=\frac{\widehat{\varphi}_{d+y}(t, t z)}{\widehat{\varphi}_{d}(t, t z)} .
$$

In particular the law $\mu$ of $\operatorname{rad}\left(\left\{\frac{x_{s}^{t}}{t}, s \in[0,1]\right\}\right)$ satisfies, for any $y \in \mathfrak{t} \oplus i \mathfrak{t}$,

$$
\int_{A} \frac{\widehat{\varphi}_{d+y}(t, t z)}{\widehat{\varphi}_{d}(t, t z)} d \mu(z)=\mathbb{E}\left(e^{\left(x_{1}^{t}, y\right)}\right)=e^{\frac{t}{2}(y, y)} .
$$

The fact that $\left|c h_{\mu}\left(e^{z}\right)\right| \leq \frac{h(\mu+\rho)}{h(\rho)}$ imply that

$$
z \in A \mapsto \frac{\widehat{\varphi}_{d}(t, z t)}{\pi(z) e_{t}(z)},
$$

where $e_{t}(z)=\sum_{\gamma \in v\left(Q^{\vee}\right)} e^{-t\left(\gamma(z)+\frac{1}{2}(\gamma, \gamma)\right)}$, is smooth on $A$. Thus the Peter-Weyl theorem ensures that for any smooth function $u$ defined on $A$, and $z \in A$,

$$
u(z) \frac{\widehat{\varphi}_{d}(t, z t)}{\pi(z) e_{t}(z)}=\sum_{\lambda \in P_{+}} c_{\lambda} \operatorname{ch}_{\lambda}(z)
$$

where $c_{\lambda}=\int_{A} u(z) \frac{\widehat{\varphi}_{d}(t, z t)}{\pi(z) e_{t}(z)} \operatorname{ch}_{\lambda}(z) \pi^{2}(z) d z$, and the convergence holds uniformly and absolutely. Actually $\lim _{|\lambda| \rightarrow \infty}(\lambda, \lambda)^{n} c_{\lambda}=0$ for all $n \in \mathbb{N}$. As $\frac{\widehat{\varphi}_{d}(t, z t)}{\pi(z) e_{t}(z)}$ remains positive on $A$,

$$
u(z)=\sum_{\lambda \in P_{+}} c_{\lambda} \operatorname{ch}_{\lambda}(z) \frac{\pi(z) e_{t}(z)}{\widehat{\varphi}_{d}(t, z t)}
$$

As $\left|\operatorname{ch}_{\lambda}(z)\right| \leq \frac{h(\lambda+\rho)}{h(\rho)}$ and $\pi(\cdot) e_{t}(\cdot) / \widehat{\varphi}_{d}(t, t \cdot)$ is bounded on $A$, the uniform and absolute convergence imply that for any probability measure $v$ on $A$,

$$
\int_{A} u(z) v(d z)=\sum_{\lambda \in P_{+}} c_{\lambda} \int_{A} \operatorname{ch}_{\lambda}(z) \frac{\pi(z) e_{t}(z)}{\widehat{\varphi}_{d}(t, z t)} v(d z) .
$$

Expression (10) for the definition of $\widehat{\varphi}_{d+y}$ gives

$$
\widehat{\varphi}_{d+2 \pi \frac{i}{t}(\lambda+\rho)}(t, t z)=\frac{1}{\pi\left(-2 \pi \frac{i}{t}(\lambda+\rho)\right)} \operatorname{ch}_{\lambda}(z) \pi(z) e_{t}(z),
$$

for any $\lambda \in P_{+}$. Thus one has

$$
\begin{aligned}
\int_{A} \operatorname{ch}_{\lambda}(z) \frac{\pi(z) e(z)}{\widehat{\varphi}_{d}(t, z t)} d \mu(z) & =\pi\left(-2 \pi \frac{i}{t}(\lambda+\rho)\right) \int_{A} \frac{\widehat{\varphi}_{d+2 \pi \frac{i}{t}(\lambda+\rho)}(t, t z)}{\widehat{\varphi}_{d}(t, t z)} d \mu(z) \\
& =\pi\left(-2 \pi \frac{i}{t}(\lambda+\rho)\right) e^{-\frac{t}{2}(2 \pi)^{2}(\lambda+\rho, \lambda+\rho)},
\end{aligned}
$$


and identity (11) becomes for $v=\mu$,

$$
\int_{A} u(z) d \mu(z)=\sum_{\lambda \in P_{+}} c_{\lambda} \pi\left(-2 \pi \frac{i}{t}(\lambda+\rho)\right) e^{-\frac{t}{2}(2 \pi)^{2}(\lambda+\rho, \lambda+\rho)} .
$$

Identity (11) becomes for $v=\mu_{u, x}$

$$
\int_{A} u(z) d \mu_{u, x}(z)=\sum_{\lambda \in P_{+}} c_{\lambda} \int_{A} \operatorname{ch}_{\lambda}(z) \frac{\pi(z) e(z)}{\widehat{\varphi}_{d}(t, z t)} d \mu_{u, x}(z) .
$$

As

$$
\int_{A} \operatorname{ch}_{\lambda}(z) \frac{\pi(z) e(z)}{\widehat{\varphi}_{d}(t, z t)} d \mu_{u, x}(z)=\pi\left(-2 \pi \frac{i}{t}(\lambda+\rho)\right) \int_{A} \frac{\widehat{\varphi}_{d+2 \pi \frac{i}{t}(\lambda+\rho)}(t, t z)}{\widehat{\varphi}_{d}(t, t z)} d \mu(z),
$$

Lemma 7.2 implies that

$$
\int_{A} u(z) d \mu_{u, x}(z) \quad \text { converges towards } \int_{A} u(z) d \mu(z)
$$

as $(u, x)$ goes to 0 within the affine Weyl chamber, which proves the proposition.

COROLlary 7.5. For any $t>0$,

$$
\lim _{(u, x) \rightarrow 0} \mathbb{Q}_{u, x}\left(b_{t} \in d z\right)=C_{t} \widehat{\varphi}_{d}(t, z) \pi\left(\frac{z}{t}\right) 1_{A}\left(\frac{z}{t}\right) \mathbb{W}_{0}\left(b_{t} \in d z\right),
$$

when $(u, x)$ goes to 0 within the affine Weyl chamber.

PROOF. The corollary follows from the Weyl integration formula which implies that the density of the radial part of $\frac{1}{t} x^{t}$ is equal to $p_{1}^{\frac{1}{t}}(z) \pi(z)^{2} 1_{A}(z)$, up to a multiplicative constant.

One defines a law $\mathbb{Q}_{0,0}$ on $\sigma\left(b_{u}: u>0\right)$ letting for $B \in \sigma\left(b_{u}: u \geq t\right), t>0$,

$$
\mathbb{Q}_{0,0}(B)=\mathbb{P}_{0}\left(C_{t} \widehat{\varphi}_{d}\left(t, b_{t}\right) \pi\left(\frac{b_{t}}{t}\right) \mathbb{Q}_{x_{t}}\left(\theta_{t} B\right)\right),
$$

where $\left\{\theta_{t}, t \geq 0\right\}$ is the shift operator.

REMARK 7.6. Under $\mathbb{Q}_{0,0}, b_{t}$ is equal in law to $\operatorname{rad}\left(t \Lambda_{0}+x^{t}\right)$ for any $t>0$. Thus for any $t>0, \frac{b_{t}}{t}$ is equal in law to $\operatorname{rad}\left(x^{\frac{1}{t}}\right)$. As $\varepsilon\left(x^{\frac{1}{t}}\right)_{1}$ converges toward the Haar measure on $K$ when $t$ goes to 0 , one obtains by the Weyl integration formula that

$$
\lim _{t \rightarrow 0} \mathbb{Q}_{0,0}\left(\frac{b_{t}}{t} \in d x\right)=C \pi(x)^{2} 1_{A}(x) d x,
$$

which can be also deduced from (12). 
REMARK 7.7. Notice that identity (8) remains true for $(u, x)=(0,0)$, with the convention of Lemma 7.1.

We are now in position to enounce the main theorem 3.1 in a more precise way. In the following, one considers a continuous version of the doubly indexed process $\left\{\varepsilon\left(t \Lambda_{0}+x^{t}\right)_{s}, s \in[0,1], t \geq 0\right\}$ (see, for instance, [4] and references therein for the existence of a continuous version). Proposition 7.9 is fundamental for the proof.

THEOREM 7.8. Let $\left\{x_{s}^{t}, s \in[01], t \in \mathbb{R}_{+}\right\}$be a standard Brownian sheet. Un$\operatorname{der} \mathbb{Q}_{0,0}$, the process $\left\{\left(t, b_{t}\right), t \geq 0\right\}$ is equal in law to the process $\left\{\left(t, \operatorname{rad}\left(t \Lambda_{0}+\right.\right.\right.$ $\left.\left.\left.x^{t}\right)\right), t \geq 0\right\}$.

ProOF. Applying the criterion given by Rogers and Pitman in Theorem 2 of [15], Proposition 7.9 gives that $\left\{\operatorname{rad}\left(x^{t}+t \Lambda_{0}\right), t \geq 0\right\}$ is a Markov process with semigroup $\left\{Q_{t}, t \geq 0\right\}$ starting from 0 .

Proposition 7.9. Let $\left\{x_{s}^{t}, s \in[0,1], t \geq 0\right\}$ be a standard Brownian sheet on $\mathfrak{k}$. Let $\Lambda$ be a kernel on $\widehat{\mathcal{C}} \times\left(\mathbb{R}_{+} \times C([0,1], \mathfrak{k})\right.$, such that for any $(t, y) \in$ $\widehat{\mathcal{C}}, \Lambda((t, y),(t, \cdot))$ is the law of a Brownian motion $\left\{x_{s}^{t}, s \in[0,1]\right\}$ on $\mathfrak{k}$, given $\operatorname{rad}\left(x^{t}+t \Lambda_{0}\right)=y$. Let $\left\{Q_{t}, t \geq 0\right\}$ be the transition probability of the Markov process $\left\{\left(t, b_{t}\right), t \geq 0\right\}$ under $\mathbb{Q}_{0,0}$, and $\left\{P_{t}, t \geq 0\right\}$ the transition probability of $\left\{\left(t, x^{t}\right), t \geq 0\right\}$. Then for any $t \geq 0$ and $(u, x) \in \widehat{\mathcal{C}}$, one has the intertwining

$$
Q_{t} \Lambda((u, x), \cdot)=\Lambda P_{t}((u, x), \cdot) .
$$

PROOF. It is sufficient to prove that for every measurable function $y \in$ $L_{2}([0,1], \mathfrak{k})$, and $r \in(0,1)$,

$$
\int Q_{t} \Lambda((u, x),(u+t, d z)) e^{\int_{0}^{r}\left(y_{s}, d z_{s}\right)}=\int \Lambda P_{t}((u, x),(u+t, d z)) e^{\int_{0}^{r}\left(y_{s}, d z_{s}\right)} .
$$

Let $y \in L_{2}([0,1], \mathfrak{k}), r \in(0,1), h \in H^{1}([0,1], K)$ such that $h^{-1} h^{\prime}=y$, and $a \in \mathfrak{t}$ such that $h_{r} \in \mathcal{O}_{e^{a}}$. On the one hand, one has

$$
\begin{aligned}
\int Q_{t} & \Lambda((u, x),(t+u, d z)) e^{\int_{0}^{r}\left(y_{s}, d z_{s}\right)} \\
& =\int Q_{t}((u, x),(u+t, d z)) \Lambda((u+t, z),(u+t, d \tilde{z})) e^{\int_{0}^{r}\left(y_{s}, d \tilde{z}_{s}\right)} \\
& =\int Q_{t}((u, x),(u+t, d z)) e^{\frac{1}{2}(u+t) \int_{0}^{r}\left(y_{s}, y_{s}\right) d s-\frac{1}{2}(t+u)(a, a)} \frac{\widehat{\varphi}_{d+a}(u+t, z)}{\widehat{\varphi}_{d}(u+t, z)} \\
& =e^{\frac{1}{2}(u+t) \int_{0}^{r}\left(y_{s}, y_{s}\right) d s-\frac{1}{2}(u+t)(a, a)} \mathbb{E}_{\mathbb{Q}_{u, x}}\left(\frac{\widehat{\varphi}_{d+a}\left(u+t, b_{t}\right)}{\widehat{\varphi}_{d}\left(u+t, b_{t}\right)}\right) \\
& =e^{\frac{1}{2}(u+t) \int_{0}^{r}\left(y_{s}, y_{s}\right) d s-\frac{1}{2} u(a, a)} \frac{\widehat{\varphi}_{d+a}(u, x)}{\widehat{\varphi}_{d}(u, x)}
\end{aligned}
$$


On the other hand,

$$
\begin{gathered}
\int \Lambda((u, x),(u, d z)) P_{t}((u, z),(u+t, d \tilde{z})) e^{\int_{0}^{r}\left(y_{s}, d \tilde{z}_{s}\right)} \\
=e^{\frac{t}{2} \int_{0}^{r}\left(y_{s}, y_{s}\right) d s} \int \Lambda((u, x),(u, d z)) e^{\int_{0}^{r}\left(y_{s}, d z_{s}\right)} \\
=e^{\frac{t}{2} \int_{0}^{r}\left(y_{s}, y_{s}\right) d s} e^{\frac{u}{2} \int_{0}^{r}\left(y_{s}, y_{s}\right) d s-\frac{1}{2} u(a, a)} \frac{\widehat{\varphi}_{d+a}(u, x)}{\widehat{\varphi}_{d}(u, x)} .
\end{gathered}
$$

Acknowledgments. This paper is dedicated to Professor Philippe Bougerol to thank him for many discussions which have been a great source of joy and inspiration to me, and a valuable aid especially in the work presented here.

\section{REFERENCES}

[1] Baudoin, F. (2002). Conditioned stochastic differential equations: Theory, examples and application to finance. Stochastic Process. Appl. 100 109-145. MR1919610

[2] BRÖCKER, T. and DIECK, T. (1985). Representations of Compact Lie Groups. Graduate Texts in Mathematics 98. Springer, New York. MR0781344

[3] Driver, B. K. (1994). A Cameron-Martin type quasi-invariance theorem for pinned Brownian motion on a compact Riemannian manifold. Trans. Amer. Math. Soc. 342 375-395. MR1154540

[4] Driver, B. K. and SRimurthy, V. K. (2001). Absolute continuity of heat kernel measure with pinned Wiener measure on loop groups. Ann. Probab. 29 691-723. MR1849175

[5] Fegan, H. D. (1978). The heat equation on a compact Lie group. Trans. Amer. Math. Soc. 246 339-357. MR0515542

[6] Frenkel, I. B. (1984). Orbital theory for affine Lie algebras. Invent. Math. 77 301-352. MR0752823

[7] GordinA, M. (2003). Quasi-invariance for the pinned Brownian motion on a Lie group. Stochastic Process. Appl. 104 243-257. MR1961621

[8] Grabiner, D. J. (1999). Brownian motion in a Weyl chamber, non-colliding particles, and random matrices. Ann. Inst. Henri Poincaré Probab. Stat. 35 177-204. MR1678525

[9] HaKim-DoweK, M. and LÉPIngle, D. (1986). L'exponentielle stochastique des groupes de Lie. In Séminaire de Probabilités, XX, 1984/85. Lecture Notes in Math. 1204 352-374. Springer, Berlin. MR0942031

[10] Helgason, S. (1989). Groups and geometric analysis. Integral geometry, invariant differential operators and spherical functions. Bull. Amer. Math. Soc. (N.S.) 20 252-256.

[11] Humphreys, J. E. (1990). Reflection Groups and Coxeter Groups. Cambridge Studies in Advanced Mathematics 29. Cambridge Univ. Press, Cambridge. MR1066460

[12] KAC, V. G. (1990). Infinite-Dimensional Lie Algebras, 3rd ed. Cambridge Univ. Press, Cambridge. MR1104219

[13] KARANDIKAR, R. L. (1983). Girsanov type formula for a Lie group valued Brownian motion. In Seminar on Probability, XVII. Lecture Notes in Math. 986 198-204. Springer, Berlin. MR0770412

[14] Pressley, A. and Segal, G. (1986). Loop Groups. Oxford Univ. Press, New York. MR0900587

[15] Rogers, L. C. G. and Pitman, J. W. (1981). Markov functions. Ann. Probab. 9 573-582. MR0624684 
LABORATOIRE DE MATHÉMATIQUES APPLIQUÉES À PARIS 5 UNIVERSITÉ PARIS 5

45 RUE DES SAINTS PÈRES

75270 PARIS CEDEX 06

FRANCE

E-MAIL: manon.defosseux@parisdescartes.fr 